\title{
Importância da suplementação com coenzima Q10 no combate aos radicais livres obtidos na atividade física de alta intensidade: uma revisão de literatura
}

Importance of supplementation with coenzyme Q10 in combating free radicals obtained in high

intensity physical activity: a literature review

Importancia de la suplementación con coenzima Q10 en la lucha contra los radicales libres

obtenidos en la actividad física de alta intensidad: revisión de la literatura

Recebido: 09/11/2021 | Revisado: 18/11/2021 | Aceito: 21/11/2021 | Publicado: 23/11/2021

Jaqueline dos Santos Silva

ORCID: https://orcid.org/0000-0002-5189-592X

Universidade Federal de Pernambuco, Brasil

E-mail: jaqueline.jss@ufpe.br

Thedemur Fortunato Soares de Oliveira

ORCID: https://orcid.org/0000-0001-5762-0831

Universidade Salgado de Oliveira, Brasil

E-mail: thedemur@gmail.com

Maria Betânia Melo de Oliveira

ORCID: https://orcid.org/0000-0001-5188-3243

Universidade Federal de Pernambuco, Brasil

E-mail: maria.bmoliveira@ufpe.br

Sivoneide Maria da Silva

ORCID: https://orcid.org/0000-0002-6507-2609

Universidade Federal de Pernambuco, Brasil

E-mail: sivoneide.maria@ufpe.br

Caio Rodrigo Dias de Assis

ORCID: https://orcid.org/0000-0001-9441-1959

Universidade Federal de Pernambuco, Brasil

E-mail: caiodias2@hotmail.com

Hévellin Talita Sousa Lins

ORCID: https://orcid.org/0000-0001-5431-9525

Universidade Federal de Pernambuco, Brasil

E-mail: hevellyn.talita@gmail.com

Renata Pereira Lima da Silva

ORCID: https://orcid.org/0000-0001-5784-8959

Universidade Federal de Pernambuco, Brasil

E-mail: renata.limas@ufpe.br

Tainara Fernandes Dantas

ORCID: https://orcid.org/0000-0001-9623-5135

Universidade Federal de Pernambuco, Brasil

E-mail: tainarafd85@gmail.com

Karolayne Silva Souza

ORCID: https://orcid.org/0000-0003-2627-7385

Universidade Federal de Pernambuco, Brasil

E-mail: karolayne.silvasouza@ufpe.br

Milena Roberta Freire da Silva

ORCID: https://orcid.org/0000-0003-0203-4506

Universidade Federal de Pernambuco, Brasil

E-mail: milena.freire@ufpe.br

Ana Vitória Araújo Lima

ORCID: https://orcid.org/0000-0003-4606-5523

Universidade Federal de Pernambuco, Brasil

E-mail: ana.vitorialima@hotmail.com

Rafael Artur de Queiroz Cavalcanti de Sá

ORCID: https://orcid.org/0000-0002-0313-1482

Universidade Federal de Pernambuco, Brasil

E-mail: rafael.aqcavalcanti2@ufpe.br

Rodrigo Reges dos Santos Silva

ORCID: https://orcid.org/0000-0003-0141-5649

Universidade Federal de Pernambuco, Brasil

E-mail: rodrigoregesufpe@gmail.com 


\author{
Danielly Lima da Silva \\ ORCID: https://orcid.org/0000-0001-6587-5084 \\ Universidade Federal de Pernambuco, Brasil \\ E-mail: daniedx11@gmail.com \\ Elisa Santiago Pereira \\ ORCID: https://orcid.org/0000-0003-4482-301X \\ Universidade Federal de Pernambuco, Brasil \\ E-mail: elisasantiagopereira1 @gmail.com
}

\begin{abstract}
Resumo
Introdução: A atividade física com a devida moderação traz benefícios para nossa saúde. No entanto, quando em excesso provoca um desgaste físico causando vários danos à saúde como lesão muscular, formação de radicais livres, estresse oxidativo e doenças degenerativas. A Coenzima Q10 (CoQ10) é uma benzoquinona essencial a vida, e está presente em todas as células do organismo humano, sendo conhecida pelo seu papel antioxidante, o presente estudo teve como objetivo identificar a relação da suplementação da CoQ10 após intensa atividade física de diferentes modalidades esportivas. Metodologia: Foi realizado um estudo de revisão da literatura em português, inglês e espanhol nas bases eletrônicas Periódicos CAPES, Pubmed, Scientific Eletronic Library On-line (SCIELO) e Scholar Google (Google Acadêmico). Foram selecionados aqueles que atendiam aos critérios de inclusão, como critério de inclusão, os artigos deveriam abordar assuntos relacionados aos descritores já citadas. Resultado: Exercícios físicos que ultrapassam o limite fisiológico, overtraining, tendem a promover um aumento na produção de radicais livres conhecidos como Espécies Reativas de Oxigênio (EROS) no organismo, os quais quando não neutralizados podem iniciar um processo deletério nas células e tecidos, ocasionando várias doenças degenerativas. A CoQ10 é uma substância produzida pelo nosso organismo, podendo também ser adquirida de fontes exógenas. Essa apresenta potente ação antioxidante e representa um componente-chave da cadeia respiratória mitocondrial gerando energia. Conclusão: De acordo com os trabalhos estudados, observou-se que a CoQ10 apresenta potente ação antioxidante no combatendo aos radicais livres, que são formados em praticantes de atividade física intensa.
\end{abstract}

Palavras-chave: CoenzimaQ10; Antioxidante; Exercício intenso; Estresse Oxidativo.

\begin{abstract}
Introduction: Physical activity in due moderation brings benefits to our health. However, when in excess, it causes physical wear and tear, causing several damages to health such as muscle damage, formation of free radicals, oxidative stress and degenerative diseases. Coenzyme Q10 (CoQ10) is an essential benzoquinone for life, and is present in all cells of the human body, being known for its antioxidant role, the present study aimed to identify the relationship of CoQ10 supplementation after intense physical activity of different sports modalities. Methodology: A literature review study was carried out in Portuguese, English and Spanish in the electronic databases CAPES, Pubmed, Scientific Electronic Library Online (SCIELO) and Scholar Google (Google Academic). Those who met the inclusion criteria were selected, as inclusion criteria, the articles should address issues related to the aforementioned descriptors. Result: Physical exercises that exceed the physiological limit, overtraining, tend to promote an increase in the production of free radicals known as Reactive Oxygen Species (ROS) in the body, which when not neutralized can initiate a deleterious process in cells and tissues, causing various degenerative diseases. CoQ10 is a substance produced by our body and can also be acquired from exogenous sources. It has a powerful antioxidant action and represents a key component of the mitochondrial respiratory chain generating energy. Conclusion: According to the studies studied, it was observed that $\mathrm{CoQ} 10$ has a powerful antioxidant action in fighting free radicals, which are formed in intense physical activity practitioners.
\end{abstract}

Keywords: CoenzymeQ10; Antioxidant; Intense exercise; Oxidative stress.

\title{
Resumen
}

Introducción: La actividad física con la debida moderación aporta beneficios a nuestra salud. Sin embargo, cuando está en exceso, provoca un desgaste físico, provocando varios daños a la salud como daño muscular, formación de radicales libres, estrés oxidativo y enfermedades degenerativas. La coenzima Q10 (CoQ10) es una benzoquinona esencial para la vida, y está presente en todas las células del cuerpo humano, siendo conocida por su papel antioxidante, el presente estudio tuvo como objetivo identificar la relación de la suplementación con CoQ10 después de una intensa actividad física de diferentes modalidades deportivas. Metodología: Se realizó un estudio de revisión de la literatura en portugués, inglés y español en las bases de datos electrónicas CAPES, Pubmed, Scientific Electronic Library Online (SCIELO) y Scholar Google (Google Academic). Se seleccionaron los que cumplieron con los criterios de inclusión, como criterio de inclusión, los artículos deben abordar cuestiones relacionadas con los descriptores antes mencionados. Resultado: Los ejercicios físicos que superan el límite fisiológico, el sobreentrenamiento, tienden a promover un aumento en la producción de radicales libres conocidos como Especies Reactivas de Oxígeno (ROS) en el organismo, que al no ser neutralizados pueden iniciar un proceso deletéreo en células y tejidos, provocando diversas enfermedades degenerativas. La CoQ10 es una sustancia producida por nuestro cuerpo y también se puede adquirir de fuentes exógenas. Tiene una poderosa acción antioxidante y representa un componente clave de la cadena respiratoria mitocondrial generadora de energía. Conclusión: De acuerdo con los 
estudios estudiados, se observó que la CoQ10 tiene una poderosa acción antioxidante en la lucha contra los radicales libres, que se forman en los practicantes de actividad física intensa.

Palabras clave: Coenzima Q10; Antioxidante; Ejercicio intenso; Estrés oxidativo.

\section{Introdução}

A realização de exercícios físicos de baixa e média intensidade, apresentam inúmeros benefícios na prevenção e controle de doenças (Brito, 2019). Uma atividade física intensa exige do organismo do atleta uma alta carga de energia, requerendo horas de treinos, levando o sistema imunológico a desenvolver um processo de adaptação. Esse desgaste físico proveniente do exercício exaustivo pode ter como consequência a perda de proteínas musculares e inflamação, desencadeando a formação de radicais livres, e dor muscular, dessa forma possibilitando a infiltração de células do sistema imune para a reparação do tecido lesado (Pedroso, 2015).

O desequilíbrio entre a produção de EROS presente nas células e a capacidade biológica para desintoxicar ou reparar os danos causados, denominamos estresse oxidativo. Quando a produção de EROS ultrapassa sua degradação, danos são causados no DNA, proteínas e fosfolipídios dos diversos tipos de células, favorecendo o aparecimento de doenças degenerativas (Porsch, 2019). Já os radicais livres, são íons, átomos ou moléculas, instáveis que possuem um ou mais elétrons desemparelhados na sua última órbita, permitindo trocas constantes de elétrons com moléculas vizinhas (Ferreira, 2020).

A coenzima Q10 (ubiquinona ou CoQ10) é uma benzoquinona com alto poder antioxidante, essencial no processo de produção energética mitocondrial e tem se mostrado eficaz no combate aos radicais livres, bem como na prevenção de doenças, incluindo doenças cardiovasculares, neuromusculares degenerativas, câncer e diabetes (Silva, 2015). O presente estudo foi realizado com o objetivo de identificar a importância da suplementação com a CoQ10 em praticantes de atividade física, de diferentes modalidades esportivas de ambos os sexos, no combate aos radicais livres gerados pelo organismo após intensa atividade física.

\section{Metodologia}

Foi realizado um estudo de revisão da literatura em português, inglês e espanhol, nas bases eletrônicas Periódicos CAPES, Pubmed, Scientific Eletronic Library On-line (SCIELO) e Scholar Google (Google Acadêmico), buscando artigos, teses e dissertações utilizando os seguintes descritores: coenzima Q10, antioxidante, exercício intenso, estresse oxidativo, no período de 2007 a 2021. Foram selecionados aqueles que se encaixavam nos critérios de inclusão. Como critério de inclusão, os artigos deveriam abordar assuntos relacionados aos descritores já citadas, ficando como critério de exclusão as publicações que não apresentaram adesão ao tema de estudo.

\section{Resultados e Discussão}

\subsection{Mitocôndria e sua relação com a CoQ10}

As mitocôndrias são conhecidas, pela sua função central na bioenergética e no metabolismo de lipídeos e aminoácidos, nela acontece a $\beta$ oxidação as reações do ciclo de Krebs e a fosforilação oxidativa (Wallace, 2013). São chamadas de usinas de força da célula, pois produzem a maior parte da energia ou ATP exigida pela célula e são essenciais para a vida eucariótica (Annesley, 2019). A mitocôndria é composta por duas membranas, as quais abrigam a matriz mitocondrial e o espaço intermembrana. Como a membrana interna manifesta maior extensão do que a externa, ela se dobra formando cristas onde se ancoram os cinco complexos da Cadeia Transportadora de Elétrons (CTE) ou cadeia respiratória. A cadeia respiratória é constituída pelos transportadores de elétrons móveis, coenzima Q e citocromo c, em conjunto com os 
quatros complexos que realizam a oxidação do substrato para conduzir a extrusão de prótons $\left(\mathrm{H}^{+}\right)$da matriz mitocondrial para o espaço intermembranar (Silva, 2016).

Responsáveis pela manutenção da vida dos seres aeróbicos e da respiração celular (fosforilação oxidativa), as mitocôndrias também são as principais produtoras de radicais livres nos humanos e demais mamíferos (Silva, 2011). Devido a uma das suas principais funções, gerar grandes quantidades de ATP por processos oxidativos, essas organelas são consideradas indispensáveis para as células (Pereira, 2015). São encontradas na forma tubular, espalhadas no citosol próximas ao núcleo celular, retículo endoplasmático, complexo de Golgi e citoesqueleto, constituindo campos magnéticos e correntes elétricas que podem ser passados para vários elementos celulares, sendo utilizado como meio de comunicação intracelular, passando continuamente por fusão e fissão (Friesen, 2015; Murray, 2015). Segundo (Littarru apud Jacobs, 2020) a CoQ10 está presente em todas as células do organismo humano, e apresenta um papel fundamental na produção de energia mitocondrial. Ela faz parte da CTE e por isso é encontrada em grandes quantidades a nível mitocondrial. A síntese endógena da CoQ10 ocorre via ciclo do mevalonato, tendo como produto final o colesterol, a CoQ10 e o dolicol (Oliveira, 2012).

\subsection{Fontes de CoQ10 e Farmacocinética}

Produzida endogenamente e, podendo ser adquirida da dieta ou de suplementos alimentares, a CoQ10 está presente com abundância em carnes, aves e peixes, sendo estas as fontes mais concentradas, o consumo diário desses alimentos fornece entre 2 a 20 mg (Santos, 2012). A absorção de CoQ10 de fontes exógenas é influenciada pela presença de alimentos e bebidas, ocorrendo mais precisamente no intestino delgado (Santos, 2012). As formas farmacêuticas encontradas no mercado são comprimidos, comprimidos mastigáveis e cápsulas com pó ou suspensão oleosa no seu interior (Bhagavan \& Chopra, 2007). Conforme Santos (2012) a eficácia da suplementação com esta coenzima na prevenção ao dano muscular provocado por exercícios físicos e treinamento desportivo está relacionada com sua ação antioxidante, consequentemente a uma redução do risco de lesão muscular em atletas.

A distribuição e captação, após a ingestão oral, dependem de suas características bioquímicas (Miles, 2007). Esta é ingerida na sua forma oxidada, sendo depois convertida pelos eritrócitos, à sua forma reduzida (Oliveira, 2012). Em seguida, é incorporada nos quilomícrons para ser transportada, sendo captada pelo fígado, onde é incorporada em Lipoproteína de Baixa Densidade (LDL), as quais transportam 58\% de CoQ10, diferentemente das Lipoproteínas de Alta Densidade (HDL) que transportam apenas 26\% (Oliveira, 2012). Estes autores também observaram que o primeiro pico da concentração máxima ocorria, frequentemente, após 6-8h da administração oral de formulações solúveis (Oliveira, 2012).

Segundo (Zaki apud Alvarenga, 2020) a CoQ10 é absorvida de forma lenta no intestino delgado, isso acontece devido a sua caraterística lipofílica, apresentando melhor absorção quando na sua forma Ubiquinol. Este composto é depois concentrado em diferentes locais, especificamente no baço, nas glândulas supras-renais, pulmões, rins e no miocárdio, apresentando um tempo de meia-vida, em nível plasmático, de 34h (Oliveira, 2012).

\subsection{Exercício intenso e a formação de radicais livres}

O exercício é de alta intensidade (ou pesado) quando realizado entre o Limiar Anaeróbio (LA) e a Potência Crítica (PC), existindo a presença de acidose metabólica (Wasserman, 2012). Tanto o exercício aeróbico quanto o anaeróbico promovem adaptações benéficas morfofuncionais e metabólicas no organismo, como a melhora na sensibilidade à ação de hormônios (insulina e leptina), a modulação nas concentrações plasmáticas dos hormônios GH (do inglês growth hormone, hormônio do crescimento), IGF-1 (Insulin Growth Factor 1 ou Fator de crescimento Insulina-1), testosterona e cortisol, responsáveis pela homeostase energética (Lorenzeti, 2011). No entanto, quando a prática é uma atividade aeróbica, muitos desconhecem a geração de radicais livres, que ocorre de forma espontânea pelo processo de respiração celular, cujo excesso 
pode causar várias doenças, inclusive a morte (Pinho, 2013). Os radicais livres são componentes químicos que reagem com proteínas, lipídios, carboidratos e ácidos nucléicos, oxidando-os e as fontes podem ser endógenas ou exógenas (Guimarães, 2013). De acordo com (Pingitore et al., 2015) a produção de radicais livres pela atividade física não intensa, apresenta benefícios na saúde, favorecendo a capacidade dos antioxidantes.

O processo de estresse oxidativo origina-se de um desequilíbrio entre a produção e a neutralização das substâncias oxidantes no organismo, favorecendo a presença elevada de radicais livres quando comparado a velocidade de remoção, ocasionando a oxidação de biomoléculas, provocando a perda de suas funções biológicas, levando ao dano oxidativo potencial contra células e tecidos (Barbosa, 2010). Dentre os malefícios provocados pelo estresse oxidativo, observamos a diminuição do desempenho físico, fadiga muscular, danos musculares, causando alterações no estado de treinamento e no sistema imunológico (Pinto, 2014).

\subsection{O sistema de defesa antioxidante}

Os antioxidantes são substâncias capazes de inibir a oxidação de biomoléculas (Vasconcelos, 2014). São moléculas que ajudam a reduzir os efeitos do estresse e da falta de oxigênio, gerando complexos que atenuam as reações produtoras de radicais livres (Goldfarb apud Vasconcelos, 2014). Os organismos vivos possuem mecanismos enzimáticos e não enzimáticos de proteção contra os danos oxidativos (Souza, 2020). O principal sistema de defesa antioxidante é composto por enzimas antioxidantes como a Catalase (CAT), Superóxido Dismutase (SOD), que diminuem com o passar dos anos, e, então, os ditos "radicais livres" aumentam e agem mais intensamente. E, a Glutationa Peroxidase (GPX), que depende da presença de nutrientes como o Zinco ( $\mathrm{Zn}$ ), Cobre (Cu), Manganês (Mn), Selênio (Se), proteínas e vitaminas (A, C, E, e complexo B) para sua formação (Vasconcelos, 2014). Estas enzimas são capazes de inativar os efeitos deletérios dos radicais livres sobre o organismo (Telesi \& Machado, 2008). Os antioxidantes atuam em diferentes níveis impedindo o ataque de radicais livres adquiridos através do metabolismo celular ou por fontes exógenas, sobre lipídeos, aminoácidos, dupla ligação dos ácidos graxos poli-insaturados e bases do DNA; evitando assim a formação de lesões, perda da integridade celular, e levando ao reparo das lesões causadas (Bianchi \& Antunes apud Vasconcelos, 2014).

\subsection{Efeito antioxidante da CoQ10}

A CoQ10, também conhecida como ubiquinona, é uma substância lipossolúvel similar à uma vitamina, que também atua como antioxidante na mitocôndria e nas membranas lipídicas, reagindo com radicais livres, muito parecida com à vitamina E (Pereira, 2013). Estudos recentes demonstram sua importância na resistência do DNA aos danos oxidativos, sua capacidade de transferir elétrons e sua ação antioxidante o que tem possibilitado um aumento no interesse de pesquisas com esta coenzima nos últimos anos (Kumar, 2009). Sua atuação como antioxidante acontece por meio da ativação e aumento da expressão de proteínas mitocondriais desacopladoras, um efeito antiapoptótico que resulta na redução de geração de radicais livres (Chaturvedi, 2008) (Tabela 1). 
Tabela 1. Efeitos da suplementação com CoQ10.

\section{População estudada e faixa Duração do etária \\ estudo}

21 atletas jovens do sexo 1 Mês masculino $(26+5$ anos de idade).

\section{Delineamento/ Metodologia}

Foram randomizados em dois grupos de acordo com estudo duplo-cego cruzado, suplementado com ubiquinol (200 mg / dia) ou placebo. Coleta de sangue antes e depois de um único exercício intenso (40 $\mathrm{min}$ a $85 \%$ maxHR).

Ratos Wistar machos de 1 mês. aproximadamente $200 \mathrm{~g}$, em número de 6 animais por caixa.
Os animais receberam alimento e água $a d$ libitum, foram divididos em quatro grupos de seis animais de acordo com cada tratamento, por 30 dias. Grupo 4 (G4)-(CoQ10 via oral) e L-tiroxina (via intraperitoneal).

1 mês.

O grupo de controle realizou o programa de
treinamento (placebo) o grupo experimental 1 realizou o programa de treinamento e recebeu
Principais Resultados $\quad$ Referências

Aumento significativo na Orlando, maioria dos (2018)

hematoquímicos.

A CoQ10 protegeu o musculo contra danos oxidativos provocados pela L-tiroxina.

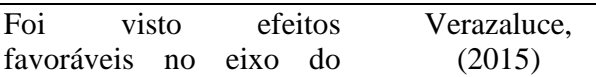
estresse ativado pelo exercício. suplementos orais de Phlebodium decumanum, posteriormente, o grupo experimental 2, além de completar o programa de treinamento físico como o resto dos grupos, tomou Phlebodium decumanum e CoQ10 em conjunto.

30 jogadores, sexo
masculino, com idade entre 22 e 32, jogadorores de vôlei, nível de treinamento alta (17 horas por semana de treinamento nos últimos 6 meses antes deste trabalho).

Homens e Mulheres 22 2x/dia em 14 Ingestão 100mg de dextrose (P) ou suplemento treinados e 19 destreinados. dias. de CoQ10 de rápida fusão, (200mg/dia). Coleta de sangue em jejum após cada teste e 2 biópsias do músculo. 70 min após ingestão do suplemento. c/ $100 \mathrm{mg}$ de 1 dos suplementos por $14 \mathrm{~d}$ e com a mesma bateria de testes.

\begin{tabular}{|c|c|}
\hline $\begin{array}{l}\text { Níveis de CoQ10 no } \\
\text { plasma } \\
\text { significativamente após } 2 \\
\text { sem de suplementação. } \\
\text { Maior tendência para } \\
\text { aumento do tempo de } \\
\text { exaustão após } 2 \text { sem. de } \\
\text { suplementação c/ CoQ10. }\end{array}$ & $\begin{array}{l}\text { Cooke, } \\
(2008)\end{array}$ \\
\hline $\begin{array}{l}\text { Diminuição na injúria } \\
\text { muscular no grupo tratado } \\
\text { com esse agente quando } \\
\text { comparado com o grupo } \\
\text { placebo. }\end{array}$ & $\begin{array}{l}\text { Kon, } \\
(2008)\end{array}$ \\
\hline
\end{tabular}

Fonte: Organizada com base nos dados dos autores.

$\begin{array}{lll}\text { Clínico randomizado duplo-cego, controlado } & \text { Diminuição na injúria } & \text { Kon, } \\ \text { por placebo. } 300 \mathrm{mg} \text { de CoQ10 por dia, } & \begin{array}{l}\text { muscular no grupo tratado } \\ \text { com esse agente quando } \\ \text { durante } 20 \text { dias ou placebo. }\end{array} & \\ & \begin{array}{l}\text { comparado com o grupo } \\ \text { placebo. }\end{array} & \\ & & \\ \end{array}$

\begin{tabular}{ll}
\hline 18 atletas de elite de kendo, & 20 dias de \\
do sexo masculino. & treinamento \\
pela manhã & $(2,5 \mathrm{~h})$ e pela \\
tarde (3h), \\
totalizando \\
5h por dia de \\
Treinamento.
\end{tabular}

A partir da análise dos artigos foi possível identificar, que a CoQ10 apresenta efeitos benéficos à saúde, atenuando os efeitos provocados pelo estresse oxidativo. O estudo feito por Cooke (2008) sobre os efeitos da suplementação aguda com CoQ10 no desempenho do exercício em indivíduos treinados e não treinados, mostrou que a suplementação aguda 2 x por 14 dias, apresenta aumento de sua concentração muscular, e diminuição do estresse oxidativo. Kon (2008) desenvolveu um estudo com um grupo de atletas de elite por 20 dias, e alcançou resultados semelhantes aos de Cooke (2008), observando diminuição na injúria muscular do grupo estudado.

Já o trabalho feito por Verazaluce (2015), demonstra que a ingestão de CoQ10 juntamente com Phlebodium decumanum (uma espécie de samambaia), é vantajosa para retardar a fadiga melhorando o desempenho esportivo e reduz o risco de sofrer lesões associadas ao exercício de alta intensididade, visto os efeitos favoráveis no eixo do estresse oxidativo. Em seu estudo a respeito do efeito da CoQ10 no músculo de ratos wistar, Silva (2015) revela que o músculo de ratos é protegido pela CoQ10 contra danos oxidativos provocados pela L-tiroxina. Entretanto, o trabalho desenvolvido por Orlando (2018), mostrou que a suplementação com a CoQ10, minimizou a depleção induzida pelo exercício uma vez que a um aumento nos níveis de antioxidantes plasmáticos e celulares, mas não foi capaz de melhorar os índices de desempenho físico ou marcadores de dano muscular. 


\section{Considerações Finais}

A CoQ10 possui uma forte ação antioxidante combatendo radicais livres, que muitas vezes são formados em praticantes de atividade física intensa. Os benefícios da coenzima são apresentados e comprovados por vários estudos, como a redução de lesão muscular, retardo na fadiga e melhora no desempenho esportivo, além do aumento de CoQ10 na concentração muscular. Contudo, se faz necessário mais estudos sobre este assunto, visto a escassez de trabalhos científicos a respeito desta temática. Por apresentar alta relevância, sugere-se que haja pesquisas futuras voltadas a CoQ10 e seu efeito nos índices de desempenho físico.

\section{Referências}

Alvarenga, L. F. (2020). Os efeitos da suplementação de coenzima q10 na terapêutica da insuficiência cardíaca: uma revisão bibliográfica. 15p. Monografia. Centro Universitário De Brasília - Uniceub.

Annesley, S. J. \& Fisher, P. R. (2019). Mitocôndrias na saúde e na doença. Células. 8(7): 680. doi:10.3390 / cells8070680.

Barbosa, K. B. F., Costa, N. M. B. \& Alfenas, R. C. G. (2010). Estresse oxidativo: conceito, implicações e fatores modulatórios. Rev. Nutr. Campinas. 23(4), $629-43$.

Bhagavan, H. \& Chopra, R (2007). Plasma coenzyme Q10 response to oral ingestion of coenzyme Q10 formulation. Mithocondrion, 7(S), S72-S88.

Bianchi, M. L. P. \& Antunes, L. M. G. (1999). Radicais livres e os principais antioxidantes da dieta. Rev Nutr. 12(2):123-30.

Brito, J. V. R., Jesus, F. M., Beserra, J. F. \& Eduardo, A. M. L. N. (2019). Suplementação De Ômega-3 Em Praticante De Exercício Físico Intenso. REVISA. $8(2): 215-27$.

Chaturvedi, R. K. \& Beal, M. F. (2008). Mitochondrial approaches for neuroprotection. Ann N Y Acad Sci.1147:395-412.

Cooke, M., Iosia, M., Buford, T. et al (2008). Effects of acute and 14-day coenzyme Q10 supplementation on exercise performance in both trained and untrained individuals. Journal of the International Society of Sports Nutrition. 5(8), 1-14.

Ferreira, J. G. S., et al (2020). Envelhecimento e a influência degenerativa dos radicais livres nesse processo. VII Congresso Internacional de Envelhecimento Humano.

Friesen, D. E., Craddock T. J., Kalra A. P., et al (2015). Biological wires, communication systems, and implications for disease. Rev. ScienceDirect. 127:14-

Goldfarb A. H. Antioxidants (1993). role of supplementation to prevent exercise-induced oxidative stress. Med Sci Sports Exerc; 25:232-6.

Guimarães, M. R. M. \& Vianna, L. M. A. (2013). Estresse oxidativo e suplementação de antioxidantes na atividade física: uma revisão sistemática. Revista Mackenzie de Educação Física e Esporte. 12(2).

Jacobs, M. A. P. \& Accursio, W. (2020). Coenzima Q10: Aplicações clínicas. BWS Journal. 3, e201100129: 1-7.

Kon, M., Tanabe, K., Akimoto, T., et al (2008). Reducing exercise-induced muscular injury in kendo athletes with supplementation of coenzyme Q10. The British Journal of Nutrition, 100(4), 903-909.

Kumar, A., Kaur, H., Devi, P. \& Mohan, V. (2009). Role of coenzyme Q10 (CoQ10) in cardiac disease, hypertension and Meniere-like syndrome. Pharmacol Ther. 124(3):259-68.

Littarru, G. P. \& Tiano, L. (2010). Clinical aspects of coenzyme Q 10: an update. Nutrition. 26:250-254. https://pubmed.ncbi.nlm.nih.gov/16205466/

Lorenzeti, F. M., Lima, W. P., Zanuto, R. et al (2011). O exercício físico modulando alterações hormonais em vias metabólicas dos tecidos musculoesquelético, hepático e hipotalâmico relacionado ao metabolismo energético e consumo alimentar. Revista Brasileira de Fisiologia do Exercício. 10(3), 172-177.

Miles, M. (2007) The uptake and distribution of coenzima Q10. Mithocondrion, 7(S), S72-S77.

Murray, A. J. \& Horscroft, J. A. (2015). Mitochondrial function at extreme high altitude. J Physiol. Epub. 10.1113/ JP270079.

Oliveira, C. I. A. (2012). Aspectos Farmácologicos Da Coenzima Q10. 85p. Monografia (Mestrado Ciências Farmaceúticas). Universidade Fernando Pessoa.

Orlando, P., Silvestri, S., Galeazzi, R., et al (2018). Efeito da suplementação de ubiquinol sobre os índices de estresse bioquímico e oxidativo após exercício intenso em jovens atletas. Redox Report. 23(1), 136-145.

Pedroso, C. O., Vicenzi, K. \& Zanette, C. (2015). Efeitos do estresse oxidativo e o uso de suplementação entre atletas. Revista Brasileira de Nutrição Esportiva. 9(53). 480-490. 
Research, Society and Development, v. 10, n. 15, e453101523056, 2021

(CC BY 4.0) | ISSN 2525-3409 | DOI: http://dx.doi.org/10.33448/rsd-v10i15.23056

Pereira, M. B. P. (2013). O papel dos antioxidantes no combate ao estresse oxidativo observado no exercício físico de musculação. Revista Brasileira de Nutrição Esportiva. 7(40), 233-245.

Pereira, B. (2015). Biogenêse mitocondrial e exercício físico: hipótese do acoplamento elétrico-transcripcional. Rev Bras Educ Fís Esporte, $17(1), 2-13$.

Pinho, W. L. \& Silva, A. P. R. (2013). Efeitos do exercício físico sobre a formação de espécies reativas de oxigênio e os compostos antioxidantes da dieta. Revista Brasileira de Nutrição Esportiva. 7(37),.77-87.

Pingitore, A., Lima, G. P., Mastorci, F., Quinones, A., Iervasi, G. \& Vassalle, C. (2015). Exercise and oxidative stress: potentialeffects of antioxidant dietary strategies in sports. Nutrition (Burbank, Los Angeles County, Calif.), 31(7-8), 916922. https://doi.org/10.1016/j.nut.2015.02.005

Pinto, E. F., Felipe, T. R., et al (2014). Avaliação do conhecimento e consumo de antioxidantes por atletas lutadores de artes marciais. Revista Brasileira de Fisiologia do Exercício. 13(3).

Porsch, L., Simas, L. A. W. \& Granzoti, R. O. C. (2019). Estresse Oxidativo E O Seu Impacto No Envelhecimento: Uma Revisão Bibliografica. Revista eletrônica. Brazilian Journal of Natural Sciences. 2(2).

Santos, R. L. (2012). Trabalho de conclusão. Curso de Habilitação em Medicina Biomolecular. Estratégia Terapêutica Regulamentada na Resolução $1500 / 1998$.

Silva, W. J. M. \& Ferrari C. K. B. (2011). Metabolismo Mitocondrial, Radicais Livres e Envelhecimento. Rev. Bras. Geriatr. Gerontol, Rio De Janeiro, 14(3):441-451.

Silva, F. B. F., Medeiros, H. C. D. Guelfi, M., et al (2015). Effect of coenzyme q10 in the 1-thyroxine-induced oxidative damage on rat soleus muscle. Rev. Bras Med Esporte. 21(2).

Silva, R. E., Larsson, N. G. \& Mourier, A. (2016). Bioenergetic roles of mitochondrial fusion. Biochimica et Biophysica Acta - Bioenergetics, 1857(8), 12771283.

Souza, L. M. V., Costa, R. A., Santos, J. D. M., et al (2020). Treinamento intervalado de alta intensidade e estresse oxidativo: uma breve apresentação. Research, Society and Development, 9(8), e 74198647, http://dx.doi.org/10.33448/rsd-v9i8.6478

Telesi, M. \& Machado, F. A. (2008). A influência do exercício físico e dos sistemas antioxidantes na formação de radicais livres no organismo humano. SaBios - Rev. Saúde e Biologia. 3(1). 40-49.

Vasconcelos, T. B., Cardoso, A., Josino, J. B. et al (2014). Radicais Livres e Antioxidantes: Proteção ou Perigo? UNOPAR Cient Ciênc Biol Saúde. 16(3):2139.

Verazaluce, J. J. G. (2015). Efecto del Phlebodium decumanum y de la coenzima q10 sobre el rendimiento deportivo en jugadores profesionales de voleibol. Nutr Hosp. 31(1):401-414.

Wallace, D. C., \& Chalkia, D. (2013). Mitochondrial DNA genetics and the heteroplasmy conundrum in evolution and disease. Cold Spring Harbor perspectives in biology, 5(11), a021220.

Wasserman, K. \& Hansen, J. E., Sue, D. Y. et al (2012). Principles of Exercise Testing and Interpretation: including pathophysiology and clinical aplications, 5th edition. Lippincott Williams \& Wilkins.

Zaki, N. M. (2016). Strategies for oral delivery and mitochondrial targeting of CoQ10. Drug Delivery. 23, 1868-1881. 